\title{
UNSTEADY NUMERICAL SIMULATIONS OF THE FLOW RELATED TO THE UNSTABLE ENERGY-DISCHARGE CHARACTERISTIC OF A MEDIUM SPECIFIC SPEED DOUBLE SUCTION PUMP
}

\author{
Olivier Braun \\ Laboratory for Hydraulic Machines - EPFL \\ Lausanne, Switzerland \\ Email: olivier.braun@epfl.ch \\ Philippe Dupont \\ Sulzer Pumps Ltd. \\ Winterthur, Switzerland \\ Email: philippe.dupont@sulzer.com
}

\author{
Francois Avellan \\ Laboratory for Hydraulic Machines - EPFL \\ Lausanne, Switzerland \\ Email: francois.avellan@epfl.ch
}

\section{ABSTRACT}

Regions of positive slope in the pressure-discharge characteristics can result in a reduction or even lack of damping of system instabilities. They are therefore one of the major concerns in design and operation of centrifugal pumps as they are limiting the admissible operating range.

The considered preliminary hydraulic design of an industrial double suction pump of medium specific speed $v=0.410$ (0.205 per impeller side) shows a marked saddle in the energydischarge characteristic associated to a sudden drop of efficiency versus discharge at part load.

Unsteady RANS type flow simulations are performed using hexahedral meshes with 2.5 million nodes to model the inlet casing, the shrouded double sided impeller with $2 \times 7$ blades, the diffuser with 12 blades and the volute.

In unsteady solution monitoring, low frequency (below blade passing frequencies) phenomena are noticed. Therefore, simulation times of up to 10 impeller revolutions at each flow rate are requested to achieve statistically steady behavior of the flow judged on global performance numbers and circumferential flow rate distribution.

The numerical simulations emphasize a drop in the characteristic, though at a lower flow rate than found on the test rig. It is shown to be associated to a one-sided separation in the diffuser, further leading to an unbalanced flow rate distribution of about $10 \%$ of flow rate between both sides of the impeller. There is a region of hysteresis, where both configurations with balanced and unbalanced flow rate distributions can be obtained for the same global flow rate.

The asymmetric flow distribution leads to asymmetric velocity profiles at the impeller-diffuser interface which results in a strongly vortical flow in the diffuser channels, where an important amount of energy is dissipated in regions of increased viscous and turbulent shear.

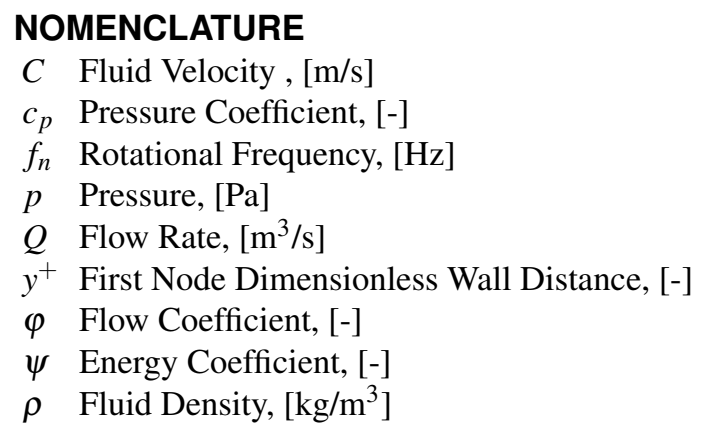


$A, B$ Side A, Side B

Loss evaluated by Area Integral of Energy Fluxes

$v$ Loss evaluated by Volume Integral of Viscous Work

Diffuser Channel i

Normalized to Best Efficiency Point

\section{INTRODUCTION}

Positive slope in the energy-discharge characteristic of centrifugal pumps has been investigated for a long time. Both experimental investigations and steady numerical simulations have shown that high specific speed impellers experience flow separations when the performance curve exhibit instability $(1,2)$. Pedersen (3) identifies alternate stall in a 2D-Pump impeller without diffuser. Former experimental and numerical studies on a medium specific pump turbine (4) have shown a flow separation on the diffuser top wall extending back into the runner to be related to an undesirable performance drop. The intense rotor-stator interaction in centrifugal pump with small gaps between impeller exit and diffuser inlet leads to highly unsteady 3-dimensional flow patterns in the rotor stator-interaction zone and the downstream diffuser that can not reasonably be treated using steady approaches $(5,6)$. Unsteady RANS approaches (7) and LES simulations (8) have both shown good predictions of unsteady pressure in turbomachinery. Moreover, a part the periodic blade passing frequencies, lower frequency phenomena like alternate stall or rotating stall in the diffuser can occur, especially at part load (9). Sano et al. relate the apparition of diffuser stall to a flat part in the diffuser performance curve and show the influence of the radial impeller-diffuser gap on the onset of different diffuser stall forms $(10,11)$. With the aim of investigating an appropriate methodology for predicting pump instability, a rather old design of a double suction pump which exhibits a performance curve with a well marked saddle shape has been selected as a case study. We first introduce the numerical method and the case study geometry and present the obtained performance curve and conclude by a comparative analysis of the simulation results of two operating points.

\section{NUMERICAL METHOD}

The flow is simulated by solving the incompressible Reynolds-averaged Navier-Stokes Equations using a commercial finite-volume solver (CFX-5) and closing the turbulent stresses by the Menter-SST-Model. The computational domain consists of block-structured hexahedral meshes containing a few prismatic elements in swept prismatic blocks to avoid low element face angles or collapsed element edges (Fig. 1). The mesh refinement is chosen based on the experience gained on former test cases (4) and with respect to the number of operating points to investigate.
Table 1. MESH SIZES PER COMPUTING DOMAIN

\begin{tabular}{cll} 
Domain & Per Passage & Total \\
\hline Inlet Casing (ic) & & $505^{\prime} 000$ \\
Impeller (a, b) & $61^{\prime} 000$ & $790^{\prime} 000$ \\
Diffuser (v) & $79^{\prime} 500$ & $824^{\prime} 000$ \\
Volute (sc) & & $417^{\prime} 000$ \\
\hline Total & & $22^{\prime} 536^{\prime} 000$
\end{tabular}

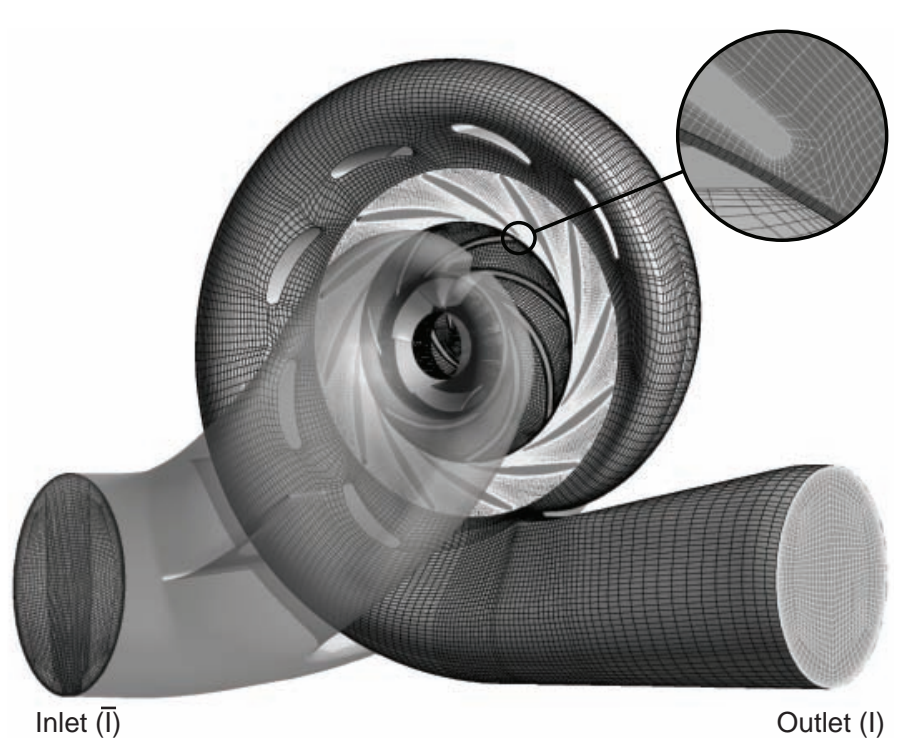

Figure 1. COMPUTATIONAL DOMAIN

The boundary conditions and interfaces represented in Fig. 1 and 2 are specified in table 2 .

Table 2. BOUNDARY CONDITIONS

\begin{tabular}{cll} 
Type & Location & Option \\
\hline Inlet & ic $(\overline{\mathrm{I}})$ & Constant Flow Rate Q \\
Outlet & sc $(\mathrm{I})$ & Zero Average Static Pressure \\
Leak. Out & $(\mathrm{a}, \mathrm{b})$ & Flow Rate $(0.8 \%$ Q per side $)$ \\
Leak. In & (ic) & Flow Rate, $\mathrm{Cu}=0.5 \mathrm{u}$ \\
Walls & - & No Slip - Log Wall Functions $\left(y^{+}=100\right)$
\end{tabular}

Copyright (c) 2007 by ASME 


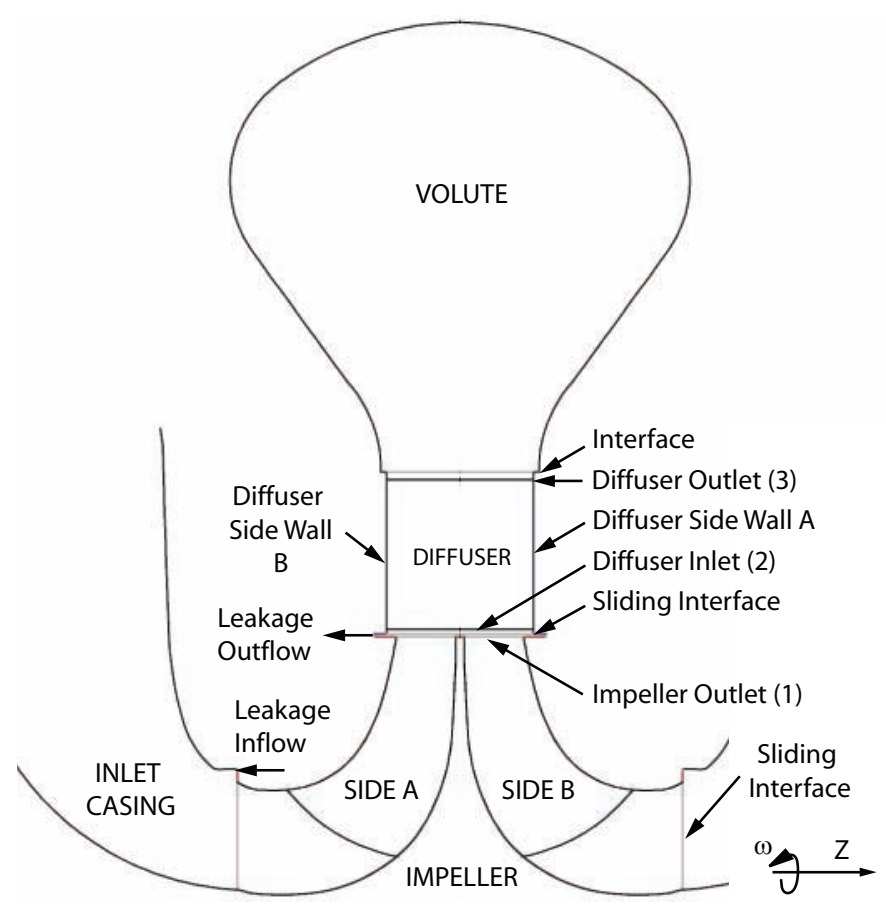

Figure 2. MERIDIONAL VIEW OF THE COMPUTING DOMAIN

For operating points below $90 \%$ of b.e.p. flow rate, low frequency fluctuations of global and local values are observed. Besides the energy coefficient $\psi$ the normalized flow coefficients of the diffuser channels v1 to v12 (Fig. 3) are used for a convergence and iteration error study:

$$
\varphi_{v i}^{*}(t)=Q_{v i}(t) \frac{z_{v}}{Q_{b e p}}
$$

At $80 \%$ flow rate, results on one revolution are obtained using different numbers of time steps $n_{t s}$ and different convergence criteria leading to $n_{c l i}$ internal coefficient loop iterations. Table 4 shows the obtained averaged energy coefficient on the last blade passage and the cumulated RMS-Error of the $\varphi_{v i}^{*}(t)$ compared to the finest one $\left(n_{t s}=840\right)$.

$$
\Sigma \Delta \varphi_{R M S}^{*}=\frac{1}{n_{t s} z_{v}} \sqrt{\sum_{i=1}^{i=z_{v}} \sum_{j=1}^{j=n_{t s}}\left(Q_{v i}\left(t_{j}\right)-Q_{v i 840}\left(t_{j}\right)\right)^{2}}
$$

\section{RESULTS}

The characteristic curve obtained with decreasing flow rate shows a sudden drop of energy coefficient at a flow rate of $75 \%$.

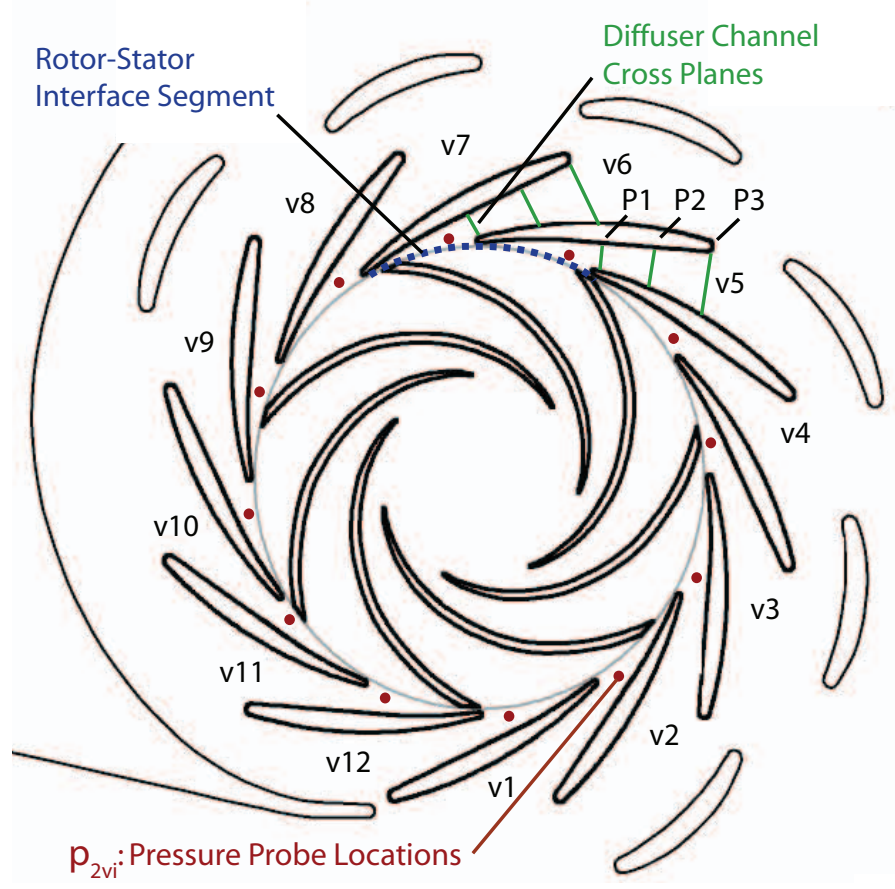

Figure 3. TOP VIEW OF THE COMPUTING DOMAIN

Table 3. TIME STEPS AND CONVERGENCE CRITERIA

\begin{tabular}{lllll}
$n_{t s}$ & RMS Res. & $n_{c l i}$ & $\psi^{*}$ & $\Sigma \Delta \varphi_{R M S}^{*}$ \\
\hline 840 & $5.10^{-6}$ & 7 & 1.095 & - \\
420 & $1.10^{-5}$ & 7 & 1.095 & 0.029 \\
$\mathbf{4 2 0}$ & $\mathbf{1 . 1 0}^{-4}$ & $\mathbf{5}$ & $\mathbf{1 . 0 9 4}$ & $\mathbf{0 . 0 3 0}$ \\
420 & $5.10^{-4}$ & 4 & 1.091 & 0.038 \\
210 & $1.10^{-4}$ & 5 & 1.085 & 0.103
\end{tabular}

The magnitude of the drop in energy coefficient is about $4 \%$. This corresponds well to the performance discontinuity measured on the preliminary design before it was cured by an oblique trim of the impeller blade trailing edge. When leading the unsteady simulations towards higher flow rates again, it reaches the performance numbers obtained downwards at $85 \%$ flow rate, a similar hysteresis has formerly been described concerning flow separation in the impeller (1).

In the following, the flow fields obtained at $80 \%$ flow rate on the downwards branch (S) of the curve will be presented and compared to the simulation results on the upwards branch (U).

At operating point $S$, the diffuser channel flow rates evaluated on 2 impeller revolutions (Fig. 5a) show a quasi-steady pat- 


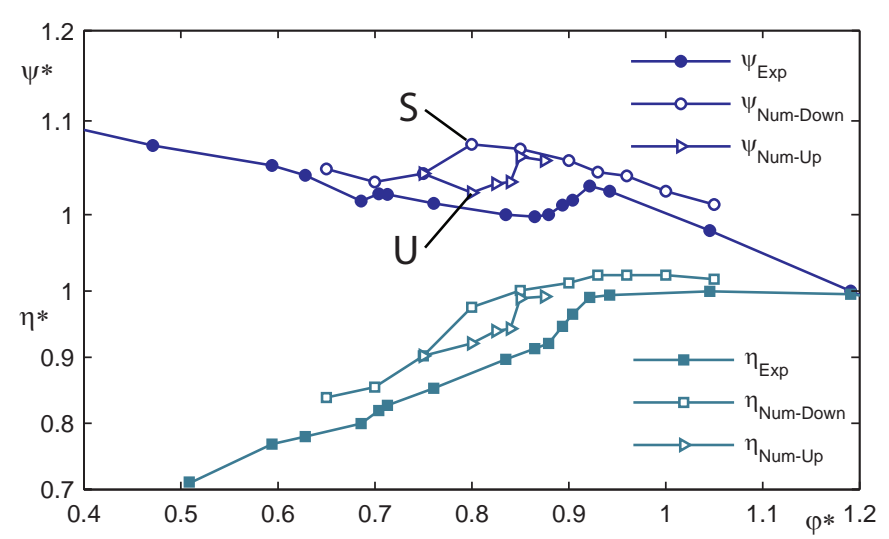

Figure 4. DIMENSIONLESS DOUBLE SUCTION PUMP HYDRAULIC CHARACTERISTIC

a

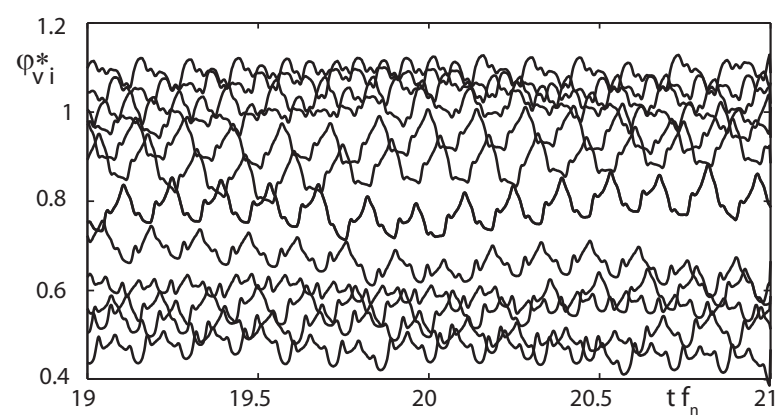

b
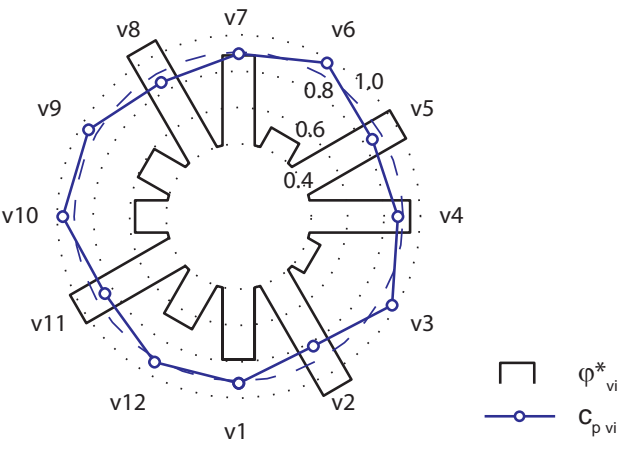

Figure 5. CIRCUMFERENTIAL FLOW RATE DISTRIBUTION AND PRESSURE PROBES AT 80\% FLOW RATE

tern of diffuser channels operating at about best efficiency point flow rate alternating with channels that are stalled at about $50 \%$ of flow rate. The pattern is not perfectly regular as in the findings of Sano et al. $(10,11)$ due to the presence of a spiral casing with the tongue and additional stay vanes that create a nonuniform pressure field at the diffuser outlet.

An experimental validation as it was performed in $(10,11)$ can be employed to asses the predictive capabilities of the numer- ical method as the time-averaged pressure on the pressure probe locations are inversely correlated to the impeller flow rates (Fig. $5 b)$.
$\varphi^{*}=0.80-S$
C Normal
(Contour $05 \quad$ 1)
-25.00
-22.50
-20.00
-17.50
-15.00
-12.50
-10.00
-7.50
-5.00
-2.50
-0.00
-2.50
-5.00
-7.50
-10.00
[m s -1$]$

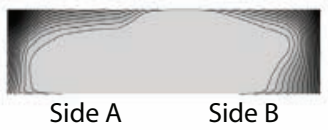

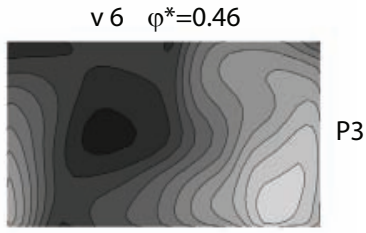
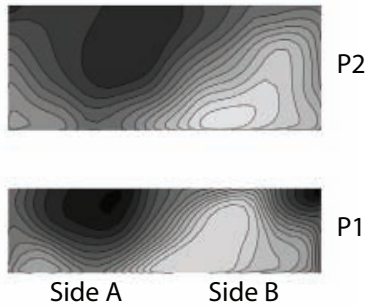

Figure 6. NORMAL VELOCITY ON DIFFUSER CHANNEL CROSS PLANES
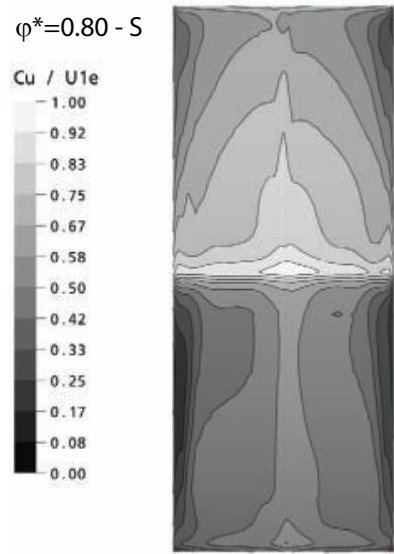

Side $\mathrm{A} \quad$ Side $\mathrm{B}$

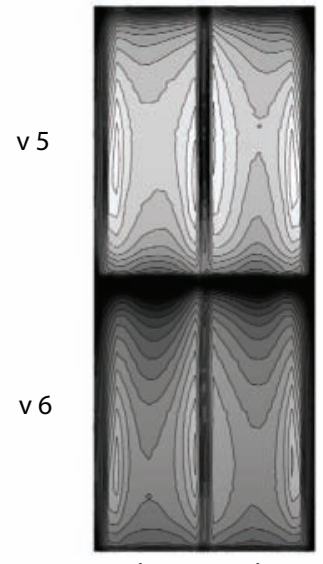

Side A Side B
Figure 7. TIME AVERAGED VELOCITIES AT DIFFUSER INLET (2)

The velocity fields in the neighboring channels v5 and v6 are different as shown in Fig. 6 and whereas the channel v5 at high flow rate has a symmetric velocity field evolution, the flow in channel v 6 is asymmetric, there is a large recirculation zone developing near the diffuser side wall A. Nevertheless, the corresponding time averaged radial and circumferential velocities in 
stationary frame at diffuser inlet (2), Fig. 7, are well symmetric, the separated flow zone does not extend into the rotating domain.
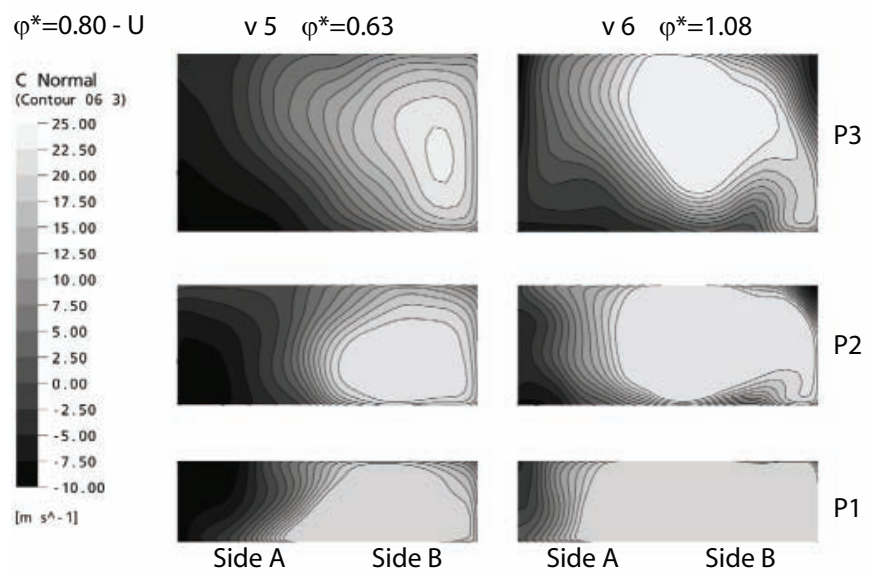

Figure 8. NORMAL VELOCITY ON DIFFUSER CHANNEL CROSS PLANES
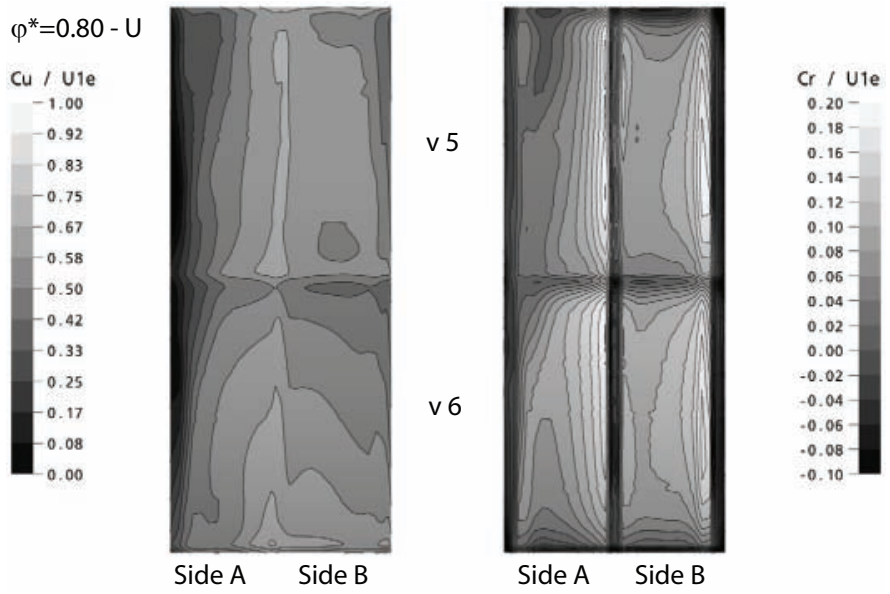

Figure 9. TIME AVERAGED VELOCITIES AT DIFFUSER INLET (2)

While the alternated pattern of channels with higher and lower flow rates is still present in $\mathrm{U}$ as in $\mathrm{S}$, the asymmetry of the separated flow in the diffuser channels is more pronounced (Fig. 8) and propagates upstream into the impeller. The impeller exit profiles show that the flow on impeller side A has drastically changed (Fig. 9).

The flow resulting from the asymmetric velocity profiles at the diffuser inlet shows a vortical structure depicted by stream- lines based on time-averaged velocity fields of one impeller revolution (Fig. 10).

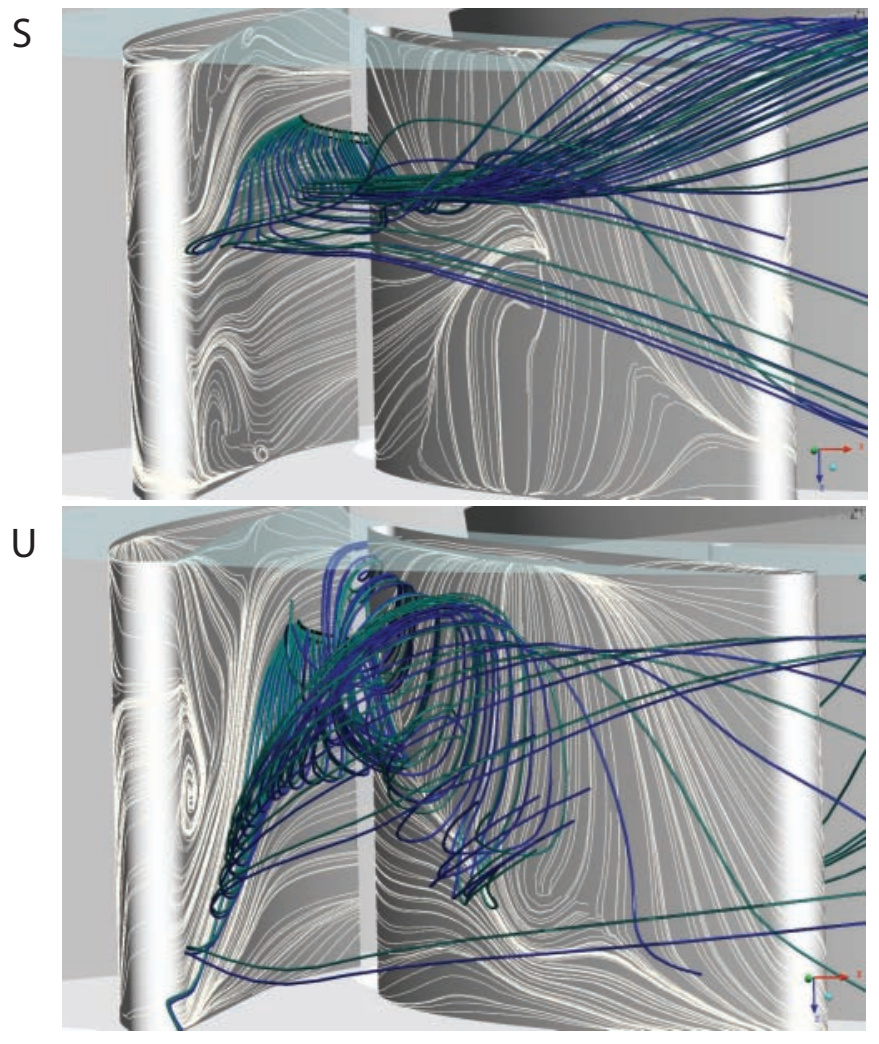

Figure 10. STREAMLINE VISUALIZATION IN THE DIFFUSER CHANNEL V7 AT 80\% FLOW RATE - SEEN FROM THE VOLUTE

An analysis of energy transfer of the pump in two flow configurations reveals that the main difference are additional losses in the diffuser domain $\mathrm{v}$ which are computed by an energy balance over one impeller revolution:

$$
\psi_{r 2 \div 3}=\frac{1}{0.5 \rho U_{1 e}^{2} Q} \frac{1}{n_{t s}} \sum_{i=1}^{i=n_{t s}} \int_{A_{3}, A_{2}} g H c_{n} d A
$$

The instantaneous viscous and turbulent losses are obtained by the volumetric integral:

$$
\psi_{r v}=\frac{1}{0.5 \rho U_{1 e}^{2} Q} \int_{v}(S: \nabla c) d v
$$

The analysis of these different terms for one impeller revolution reveals that the additional losses occurring for asymmetric 
flow are located in the diffuser and the spiral casing. The analysis of the volumetric viscous loss term does not reproduce the entire losses which indicates a lack of conservation of kinetic energy in the numerical method. The difference in diffuser losses between both flow configuration is well captured in the volumetric term.

Table 4. ENERGY TRANSFER IN THE PUMP AT 80\% FLOW RATE

\begin{tabular}{clll} 
Value & S-Symmetric & U - Asymmetric & Difference U-S \\
\hline$\psi^{*}$ & 1.094 & 1.037 & 0.056 \\
$\psi_{2}^{*}$ & 1.216 & 1.212 & 0.004 \\
$\psi_{r 2 \div 3}^{*}$ & 0.104 & 0.140 & 0.036 \\
$\psi_{r v 2 \div 3}^{*}$ & 0.046 & 0.077 & 0.031 \\
$\psi_{r 3 \div I}^{*}$ & 0.019 & 0.044 & 0.025
\end{tabular}

Furthermore the normalized impeller side flow rates $\varphi_{A}^{*}$ and $\varphi_{B}^{*}$ are diverging towards a difference of about 0.1 when the asymmetrically detached diffuser flow occurs.

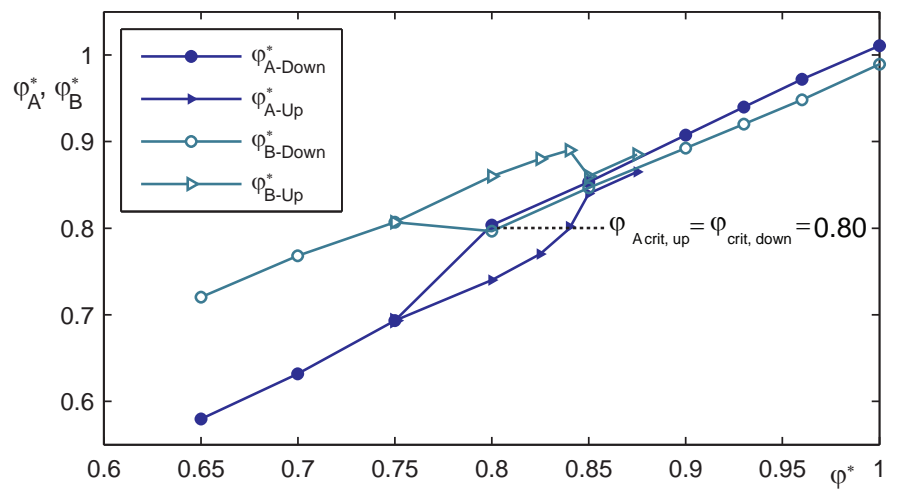

Figure 11. DIMENSIONLESS FLOW RATE BALANCE BETWEEN THE TWO IMPELLER SIDES

The same hysteresis than for global performance number is observed for the flow rate repartition. The flow separation and the discharge imbalance vanish with increasing flow rates when the lower of the two impeller side flow rates reaches the same critical discharge as when the separation first occurs on decreasing flow rates.

Pressure Values for 24 probe points $p_{v i A}$ and $p_{v i B}$ are extracted from the numerical results for three impeller revolutions for the operating points U and S. The Fourier Series decomposition is computed from the each of signals. The time average values on side A are slightly higher than side B for the asymmetric flow condition (higher pressure on the separated flow side). The more revealing phenomenon is the frequency content of the signals. Whereas the harmonics frequencies of the blade passage frequencies are in the same order of magnitude comparing both cases $\mathrm{U}$ and $\mathrm{S}$, there is significantly more broad band noise in the range up to 3 times blade passage frequency $f_{n}$ in the case $\mathrm{U}$ of asymmetric separated diffuser flow, especially on the separated flow side A of the diffuser (Fig. 12).
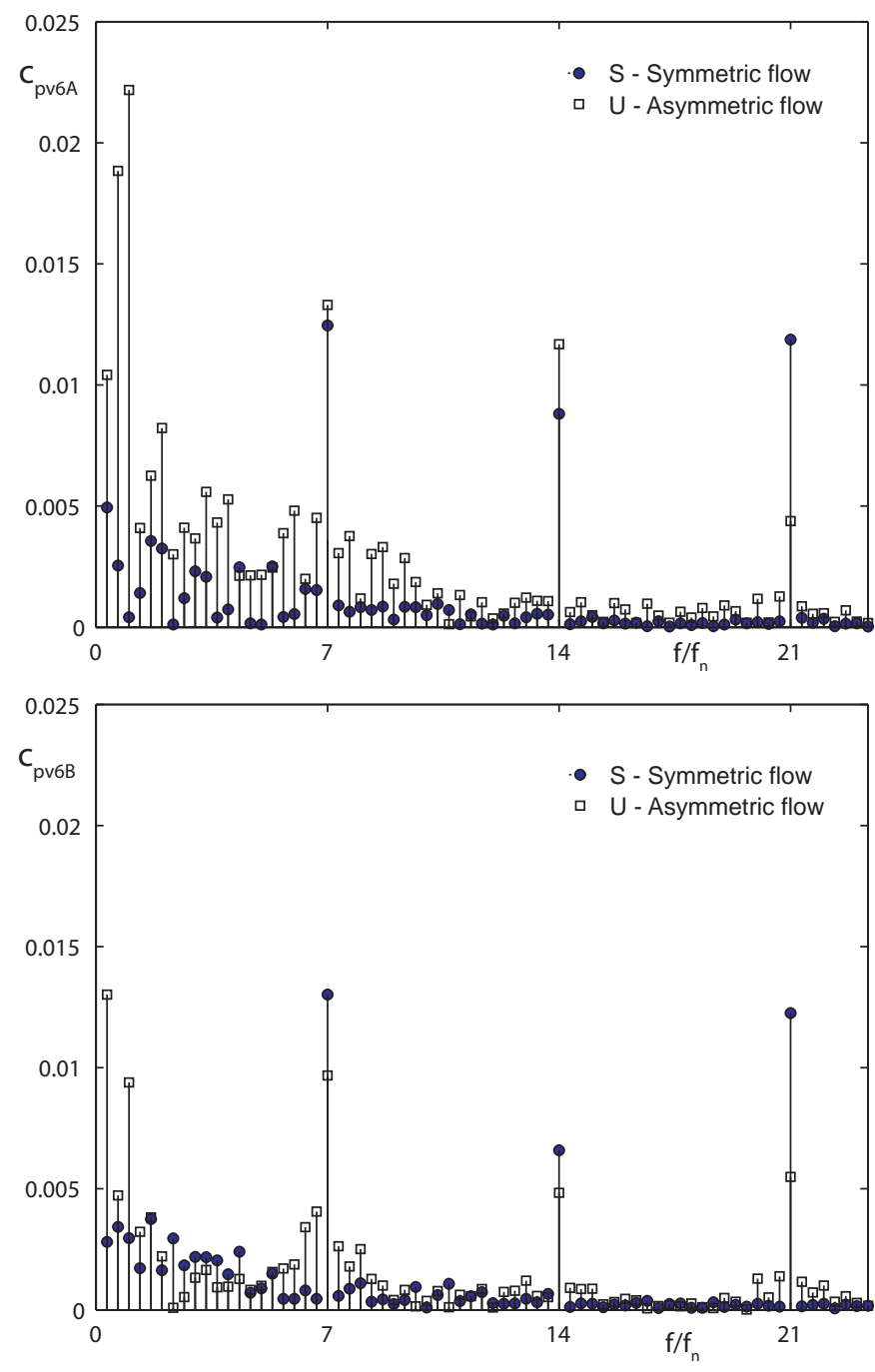

Figure 12. PRESSURE AMPLITUDE SPECTRA AT DIFFUSER CHANNEL V6 INLET AT 80\% FLOW RATE ON DIFFUSER SIDE A AND B 
The broad band amplitudes are about $0.2 \%$ to $0.5 \%$ of the pump head, compared to $1.5 \%$ for the harmonics of blade passage frequencies. They can be identified with commonly used measurement and signal analysis techniques.

\section{SUMMARY AND CONCLUSIONS}

The unsteady flow simulations of an industrial double suction pump show two major governing phenomena: At operating points below $90 \%$ of best efficiency point, patterns of alternate stall occur. Below $80 \%$ of b.e.p. flow rate, a one-sided flow separation in the diffuser occurs that influences the impeller exit flow profiles and leads to a strongly vortical flow in the diffuser channel where large amounts of energy are dissipated. These losses affect the characteristic to have a positive slope. This finding is in good agreement with the characteristic measured on the test rig, though it does not appear at the same flow rate. Less diffusive turbulence models such a LES or DES can contribute to the validation of the present RANS-type numerical studies for the operating points of interest, both regarding the overall flow field and the local pressure fluctuation levels. The presented analysis of dynamic pressure differences on the opposite sides of the diffuser provides a prospect of a feasible experimental approach for the identification of asymmetric flow in radial pump diffusers.

\section{ACKNOWLEDGMENT}

This study is established within the Project 'Development of CFD procedures for improving the stability of head-capacity characteristics of turbo pumps' whose participants and financial supporters are Sulzer Pumps, the EPFL and the Swiss Federal Office for Professional Education and Technology (OPET). The authors wish to thank Sulzer Ltd. for providing the preliminary design of a rather old pump as a test case and the EPFL central computing facility cluster Mizar for the resources and their staff for the kind support in using those.

\section{REFERENCES}

[1] Kaupert, K., Holbein, P., and Staubli, T., 1996. "A first analysis of flow field hysteresis in a pump impeller". Journal of Fluids Engineering, Transactions of the ASME, 118(4), pp. 685-691.

[2] Jaberg, H., and Hergt, P., 1997. "Head curve stability in radial pumps". Proceedings of the 1997 2nd European Conference on Turbomachinery - Fluid Dynamics and Thermodynamics, Antwerpen, Belgium, 5-7 March 1997, pp. 365372.

[3] Pedersen, N., Larsen, P.-S., and Jacobsen, C.-B., 2003. "Flow in a centrifugal pump impeller at design and offdesign conditions - part i: Particle image velocimetry (piv) and laser doppler velocimetry (ldv) measurements". Journal of Fluids Engineeering, 125, January, pp. 61-72.

[4] Braun, O., Kueny, J.-L., and Avellan, F., 2005. "Numerical analysis of flow phenomena related to the unstable energydischarge characteristic of a pump-turbine in pump mode". In Proceedings of the American Society of Mechanical Engineers Fluids Engineering Division Summer Conference, Vol. 1 PART B, pp. 1075-1080.

[5] Eisele, K., Zhang, Z., Casey, M., Gülich, J., and Schachenmann, A., 1997. "Flow analysis in a pump diffuser - part 1: Lda and ptv measurements of the unsteady flow". Journal of Fluids Engineering, Transactions of the ASME, 119(4), pp. 968-977.

[6] Muggli, F., Eisele, K., Casey, M., Gülich, J., and Schachenmann, A., 1997. "Flow analysis in a pump diffuser - part 2: Validation and limitations of cfd for diffuser flows". Journal of Fluids Engineering, Transactions of the ASME, 119(4), pp. 978-984.

[7] Zobeiri, A., Kueny, J.-L., Farhat, M., and Avellan, F., 2006. "Pump-turbine rotor-stator interactions in generating mode: Pressure fluctuation in distributor channel”. In 23rd IAHR Symposium - Yokohama.

[8] Nagahara, T., Inoue, Y., Sato, T., Sakata, S., Nishimura, K., and Kato, C., 2005. "Investigation of the flow field in a multistage pump by using les". In Proceedings of the American Society of Mechanical Engineers Fluids Engineering Division Summer Conference, Vol. 1 PART B, pp. 1321-1329.

[9] Wang, H., and Tsukamoto, H., 2003. "Experimental and numerical study of unsteady flow in a diffuser pump at offdesign conditions". Journal of Fluids Engineering, Transactions of the ASME, 125(5), pp. 767-778.

[10] Sano, T., Yoshida, Y., Tsujimoto, Y., Nakamura, Y., and Matsushima, T., 2002. "Numerical study of rotating stall in a pump vaned diffuser". Journal of Fluids Engineering, Transactions of the ASME, 124(2), pp. 363-370.

[11] Sano, T., Nakamura, Y., Yoshida, Y., and Tsujimoto, Y., 2002. "Alternate blade stall and rotating stall in a vaned diffuser". JSME International Journal, Series B: Fluids and Thermal Engineering, 45(4), pp. 810-819. 